\title{
Regenerating Agent (RGTA) based matrix therapy for treatment-resistant persistent epithelial defects
}

\author{
Nilgün Solmaz*, Güldal Koca and Feyza Önder \\ Haseki Training and Research Hospital, Ophthalmology Department, Istanbul, Turkey
}

\begin{abstract}
Persistent corneal epithelial defects (PED) can lead to potentially destructive results. In longer lasting defects, stromal melting starts and perforation may develop. Therefore, epithelial defects should be aggressively treated. In recent years, regenerating agent (RGTA) based matrix therapy has been presented as a new therapeutic approach for the treatment of PED and neurotrophic ulcers. We report the successful application of RGTA (Cacicol20, Paris, France) for the post - keratoplasty PED resistant to 4 weeks of conventional therapy including $30 \%$ autologous serum and lastly temporary tarsorrhaphy. Cacicol20 was applied once every other day. The PED started to shrink in a week and healed completely in 4 weeks. No side effect was observed. RGTA seems to be an effective therapeutic agent for PED resistant to conventional therapy. However; randomized clinical trials are needed to be able to better assess the effectiveness and the safety of the treatment.
\end{abstract}

\section{Introduction}

A breach in the integrity of the corneal epithelium results in woundhealing response. The normal healing process of the corneal epithelium is quite complex, involving matrix proteins, their corresponding integrin receptors, growth factors, and numerous proteolytic enzymes [1]. The epithelial defects not healing within the normal time frame (usually defined in the literature as 2 weeks) are named as persistent epithelial defects (PED) [2].

ReGeneraTing Agents (RGTAs) are biopolymers engineered as heparan sulfate analogues. They take place of degraded heparan sulfates in the injured tissue and bind to extracellular matrix (ECM) proteins and growth factors; and protect ECM from proteolysis and reconstruct the micro-medium necessary for wound healing [3]. In in vitro conditions, in vivo cell cultures and animal models, it was shown that they inhibit various proteolytic enzymes, control inflammation, and regulate collagen synthesis [3-5]. Although clinical use is currently limited, reports of promising outcomes of RGTA use in treatment of chronic corneal ulcers and PED that are resistant to conventional treatments started to be published in the recent years [6-9].

We present the successful application of RGTA (Cacicol20, Paris, France), also called matrix therapy, for the treatment of postkeratoplasty PED resistant to conventional therapy.

\section{Case report}

Sixty-nine-year-old female patient with chronic closed angle glaucoma, who had trabeculectomy 3 years ago and intraocular pressure (IOP) was under control without any medication, underwent cataract surgery for grade III nuclear cataract. She developed pseudophakic bullous keratopathy following uncomplicated phacoemulsification surgery and penetrating keratoplasty (PK) was performed. On the postoperative $1^{\text {st }}$ day, the corneal graft was edematous and total epithelial defect was observed. Topical steroid (dexamethasone $0.1 \% 6$ times daily), topical antibiotic (lomefloxacin $0.3 \%$ eye drops, 4 times daily), topical cyclopentolate hydrochloride 1\%, 3 times daily, and nonpreserved artificial tear (sodium hyaluronate 0.15\%, 6 times daily) were initiated. The epithelial defect on the donor cornea decreased from the periphery to the center during postoperative first 3 days, and the improvement stopped when an epithelial defect of about $3.5 \times 3.0 \mathrm{~mm}$ persisted at the central cornea. On the postoperative $5^{\text {th }}$ day, the patient was switched from these medications to non-preservative forms. The frequency of administration of artificial tear drops was increased, and pressure bandage was started to be applied. On the $9^{\text {th }}$ day, autologous serum $30 \%$ (5 times daily) was added. On the $14^{\text {th }}$ day, PED diagnosis was made and temporary tarsorrhaphy was performed. No significant changes were observed in the size of the epithelial defect at the end of the conventional treatment period of 4 weeks in total (Figure 1A). The autologous serum was discontinued and RGTA-based matrix therapy (Cacicol20) was initiated. Cacicol20 was applied on alternate days, as the first eye drop of the day. It was accompanied with topical dexamethasone (Dexa-sine SE, Liba Laboratories) 4 times a day, topical moxifloxacin $0.5 \%$ (Vigamox, Alcon Laboratories) 4 times a day, and artificial tear (sodium hyaluronate $0.15 \%$ ) 6 times a day.

The shrinkage in the PED was observed in the next visit on the following week. On day 20, upon detection of significant shrinkage $(2.8 \times 1.2 \mathrm{~mm})$ (Figure 1B), tarsorrhaphy was opened and Cacicol20 treatment was combined with application of silicone hydrogel bandage contact lens (BCL, Senofilcon A, Acuvue Oasys, Johnson \& Johnson). At the control visit after one week, the epithelium defect was determined to have fully closed (Figure $1 \mathrm{C}$ and $\mathrm{D}$ ). The combined treatment was continued for one more week because of a "whorl shaped" keratopathy. No side effect, no discomfort was observed throughout the treatment. No recurrence occurred within the 12-month follow-up period.

Correspondence to: Nilgün Solmaz, Haseki Training and Research Hospital, Ophthalmology Department, Istanbul, Turkey, Tel: +90 5334210019; E-mail: nilgunsolmaz@hotmail.com

Key words: persistent epithelial defect, regenerating agent (RGTA), matrix therapy

Received: July 25, 2017; Accepted: August 22, 2017; Published: August 25, 2017 

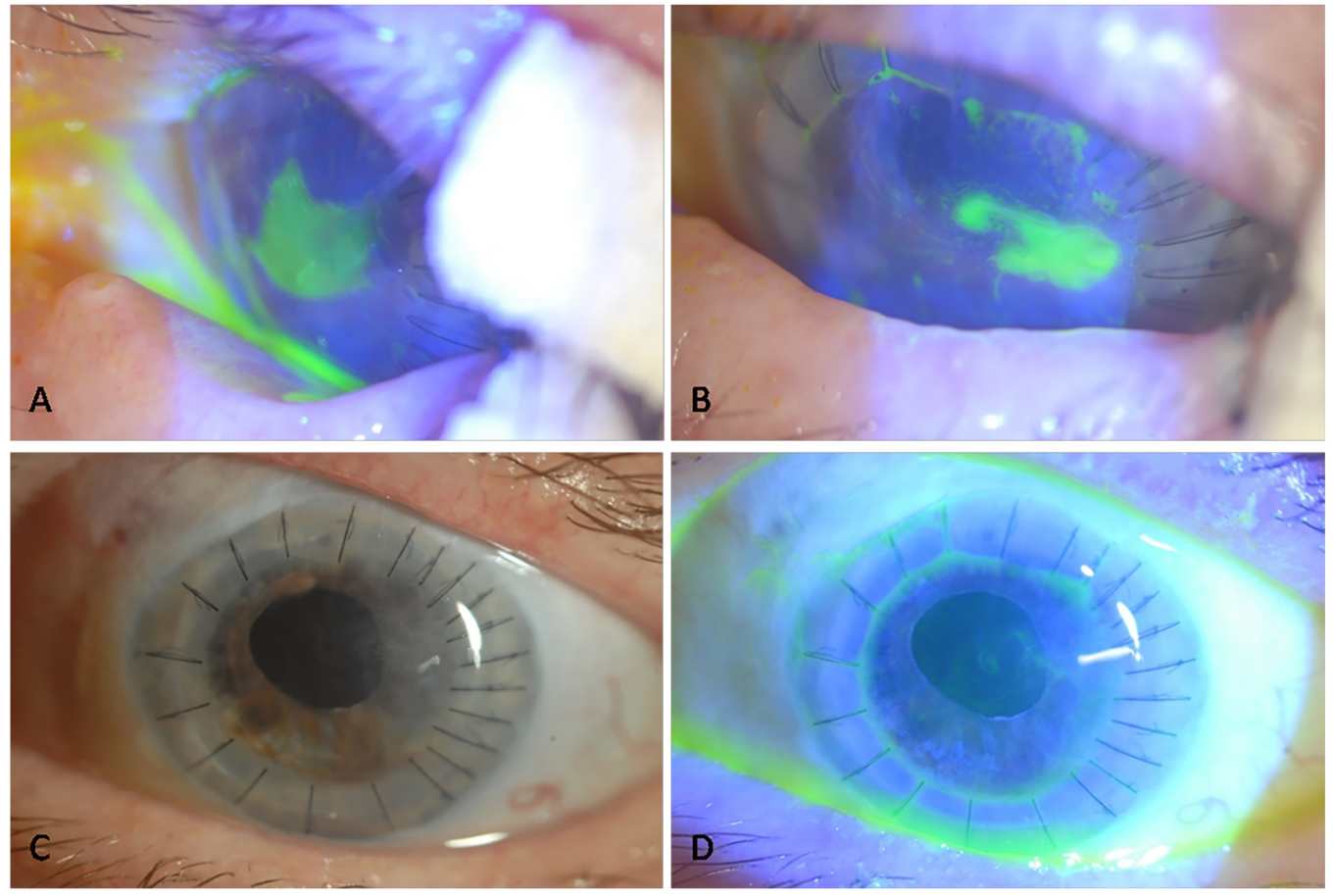

Figure 1. A) PED, $3.5 \times 3 \mathrm{~mm}$ in size that has not responded to a 4-week conventional therapy including tarsorrhaphy. B) Significant shrinkage in PED on day 20 of Cacicol20 therapy. C and D) Fully recovered PED on week 4 of treatment; and clear BCL with no deposit.

\section{Discussion}

Delayed or incomplete epithelialization of the corneal transplant can lead to potentially disastrous results. Within about 1 week, the Bowman's membrane may be damaged, and superficial haze and scar may persist on the graft even if epithelialization is subsequently completed. In longer lasting defects, stromal melting starts and perforation may develop. These also pose a risk for infections. Epithelial defects should therefore be aggressively treated, and treatment should be proceeded step by step [10]. For epithelial defects that do not disappear within a few days despite switching from topical medications to non-preservative forms, aggressive lubrication and mild pressure bandage, autologous serum and bandage contact lens should be considered; and if this fails, tarsorrhaphy should be performed [10]. Amniotic membrane transplantation (AMT) can be considered as the other treatment option. But it makes cornea difficult to be observed. In addition, corneal graft and trabeculectomy blebs can be damaged during AMT.

Our patient developed PED during the acute period after keratoplasty, and stepwise treatment was performed. Firstly, the medications were switched to non-preservative forms, and pressure bandage was applied. Since this treatment failed, autologous serum was initiated, and tarsorrhaphy was performed at the end of the $2^{\text {nd }}$ week. Since the conventional treatment period of 4 weeks in total failed, the patient was switched to RGTA therapy and resulted in complete healing.

RGTAs are bioengineered structural analogues of heparin sulfate glycosaminoglycans and are thought to mimic the function of ECM components [3]. Unlike endogenous heparin sulfate, these polymers are stable and resistant to degradation in a proteolytic environment. When applied to topically an ulcer, they penetrate into the micro-clefts of the injured ECM and replace the degraded endogenous heparin sulfate. RGTAs are specifically bound to structural matrix proteins and growth factor. The RGTA-bound proteins are protected from proteolysis, and this effects allow recreating a suitable micro-environment for cells to the tissue regeneration [6]. In recent years, various studies showed that RGTAs may be an alternative noninvasive therapeutic approach in neurotrophic keratopathy management, PED and chronic ulcers resistant conventional therapies [7-9].

Also in our case, the post-keratoplasty PED that do not respond to conventional therapy including tarsorrhaphy healed with RGTA-based matrix therapy. No signs of ocular irritation, discomfort and side effect were observed. This suggests that RGTAs may be a possible alternative therapy to surgical approach such as tarsorrhaphy or AMT in severe persistent corneal epithelial defects.

\section{Conclusion}

RGTA, which stimulates tissue regeneration by imitating the functions of ECM components, seems to be an effective and well tolerated therapeutic agent for the treatment of PED resistant to conventional therapy. However, prospective randomized clinical trials are needed to be able to better assess the effectiveness and the safety of the treatment.

\section{References}

1. Watkins A, Macalusa DC, Feldman ST (2005) Pathogenesis of sterile corneal erosions and ulcerations. In Krachmer JH, Mannis MJ, Holland EJ, editors: Cornea, 2rd edition, China, Elsevier Mosby.

2. Jeng BH (2011) Treating the nonhealing epithelial defect. Cataract Refract Surg Today Europe September: 25-28.

3. Neck JV, Tuk B, Barritault D, Tong M (2012) Heparan Sulfate Proteoglycan Mimetics Promote Tissue Regeneration: An Overview, Tissue Regeneration - From Basic Biology to Clinical Application, Prof. Jamie Davies (Ed.), InTech, DOI: 10.5772/25622. Available from: https://www.intechopen.com/books/tissue-regeneration-from-basicbiology-to-clinical-application/heparan-sulfate-proteoglycan-mimetics-thrive-tissueregeneration-an-overview

4. Cejkova J, Olmiere C, Cejka C, Trosan P, Holan V (2014) The healing of alkali-injured cornea is stimulated by a novel matrix regenerating agent (RGTA, CACICOL20): a biopolymer mimicking heparan sulfates reducing proteolytic, oxidative and nitrosative damage. Histol Histopathol 29: 457-478. [Crossref] 
5. Brignole-Baudouin F, Warnet JM, Barritault D, Baudouin C (2013) RGTA-based matrix therapy in severe experimental corneal lesions: safety and efficacy studies. $J F r$ Ohthalmol 36: 740-747. [Crossref]

6. Chebbi CK, Kichenin K, Amar N, Nourry H, Warnet JM, at al. (2008) [Pilot study of a new matrix therapy agent (RGTA OTR4120) in treatment-resistant corneal ulcers and corneal dystrophy]. J Fr Ophtalmol 31: 465-471. [Crossref]

7. Aifa A, Gueudry J, Portmann A, Delcampe A, Muraine M (2012) Topical treatment with a new matrix therapy agent (RGTA) for the treatment of corneal neurotrophic ulcers. Invest Ophthalmol Vis Sci 53: 8181-8185. [Crossref]
8. De Monchy I, Labbe A, Pogorzalek N, Gendron G, M'Garrech M, at al. (2012) Management of herpes zoster neurotrophic ulcer using a new matrix therapy agent (RGTA): a case report. J Fr Ophtalmol 35: 187. e1-e6. [Crossref]

9. Kymionis GD, Liakopoulos DA, Grentzelos MA, Diakonis VF, Klados NE, at al (2014) Combined topical application of a regenerative agent with a bandage contact lens for the treatment of persistent epithelial defects. Cornea 33: 868-872. [Crossref]

10. Levenson J (2009) Penetrating corneal transplantation: early postoperative management In Mcdonnell PJ, Mcghee CNJ, Farjo AA, Serdarevic ON, editors: Corneal Surgery, 4rd edition, China, Elsevier Mosby.

Copyright: $\subset 2017$ Solmaz N. This is an open-access article distributed under the terms of the Creative Commons Attribution License, which permits unrestricted use, distribution, and reproduction in any medium, provided the original author and source are credited. 